\title{
Japanese Encephalitis Virus Antibody Measurement
}

National Cancer Institute

\section{Source}

National Cancer Institute. Japanese Encephalitis Virus Antibody Measurement. NCI

Thesaurus. Code C147378.

The determination of the amount of Japanese encephalitis virus antibody present in a sample. 